\title{
Enseñanza de la ética en las escuelas de medicina peruanas: un estudio de sílabos
}

Ethics education in Peruvian medical schools: a study of syllabi

\author{
Maximiliano Cárdenas Díaz ${ }^{1, a}$, Cecilia Sogi Uematsu, \\ 'Instituto de Ética en Salud, Facultad de Medicina, Universidad Nacional Mayor de San Marcos. \\ ${ }^{a}$ Departamento de Medicina Preventiva y Salud Pública; ${ }^{b}$ Departamento de Siquiatría.
}

\begin{abstract}
Resumen
Objetivo: Describir las características generales de la enseñanza de ética (nombre, año de inicio del curso y organización), metodología de enseñanza y evaluación del aprendizaje, objetivos, contenidos y material de lectura, expuestas en los silabos del curso de las escuelas médicas peruanas. Diseño: Estudio descriptivo, transversal, observacional. Institución: Instituto de Ética en Salud, Facultad de Medicina, Universidad Nacional Mayor de San Marcos. Material: Sílabos de ética. Métodos: Se utilizó una fuente de información secundaria consistentes en 25 sílabos del curso de ética, de 22 escuelas de medicina: 13 públicas y 9 privadas (tres escuelas contaban con dos cursos). Se elaboró una base de datos en Excel, se utilizó el paquete estadístico SPSS versión 15 para análisis de frecuencias y Atlas ti para análisis cualitativo. Principales medidas de resultados: Características generales de la enseñanza de ética. Resultados. La denominación del curso más frecuente fue Ética y deontología. Diecinueve escuelas tenían uno y tres dos cursos de ética, y el internado médico no consideró la asignatura. La formación ética solo ocupó el 1\% (2\% en escuelas con dos cursos) del total de créditos establecidos para graduarse de médico cirujano. El método de enseñanza fue en aula y no en campo clínico y las escuelas cumplian con evaluar formalmente el aprendizaje del conocimiento. El objetivo más frecuente fue de conocimiento (43\%). Los contenidos más frecuentes se referian a profesionalismo (21\%). Los 25 sílabos listaban un total de 240 lecturas, siendo la más frecuente el código de ética del Colegio Médico del Perú. Conclusiones: Hay necesidad de llevar a cabo esfuerzos de consensos interinstitucionales y de expertos en el tema de educación en ética médica.
\end{abstract}

Palabras clave: Educación ética, silabos, caracteristicas generales, objetivos, contenidos.

\section{Abstract}

Objectives: To describe the general characteristics of medical ethics education, teaching methods and assessment, objectives, areas content and readings required as contained in Peruvian medical schools syllabi. Design: Descriptive, transversal, observational study. Setting: Institute of Ethics in Health, Faculty of Medicine, Universidad Nacional Mayor de San Marcos, Lima, Peru. Material: Ethics course syllabi. Methods: Information of 25 ethics course syllabi corresponding to 22 medical schools were used: 13 public and 9 private (three schools had two courses). Data contained in the syllabi were analyzed using SPSS version 15 and Atlas ti for qualitative analysis. Main outcome measures: Characteristics of teaching on ethics. Results: All Peruvian medical schools required ethics course in their curricula. Most frequent name was Ethics and deontology. Most schools had only one course $(n=19)$ and three had two. There was no course on ethics during internship. The course on ethics represented only $1 \%$ (2\% in schools with two courses) of total credits required for graduation as a physician in our country. The most frequent method of teaching was in the classroom and not in clinical settings. All schools assessed ethics learning through knowledge tests. The most frequent objective was knowledge (43\%) and the most frequent content area was professionalism (21\%). The 25 syllabi listed 240 readings, being the most frequent the Colegio Medico del Peru's Code of Ethics. Conclusions: There is necessity of consensus for ethics education in our medical schools.

Key words: Ethics education, syllabi, general characteristics, objectives, contents.

An Fac med. 2013;74(2):107-15

\section{INTRODUCCIÓN}

Tradicionalmente, la educación de los futuros médicos en las formas correctas de conducirse profesionalmente y de tomar decisiones que afectan la vida de otras personas, no ha sido objeto específico de curso o programa de enseñanza alguna, entre los múltiples que impartían las escuelas de medicina. Esta situación comenzó a cambiar allá por los años sesenta, específicamente en los Estados Unidos de Norteamérica. Se empezó a cuestionar la actuación del médico, en su ejercicio profesional como clínico e investigador biomédico. Tales cuestionamientos provenían tanto del propio seno de la profesión médica como de determinados sectores de la sociedad.

La reflexión, así como los estudios y debates que tuvieron lugar, suscitó, entre otros, dos acontecimientos trascen- dentales: el surgimiento de la Bioética clínica como disciplina y el inicio de la educación formal en ética en las escuelas de medicina ${ }^{(1)}$. Ambos desarrollos tienen factores comunes: la incesante innovación tecnológica en la práctica clínica, el incremento explosivo de los costos de la atención médica, las limitaciones de los recursos disponibles, la ponderación de la calidad de vida, la afirmación de los derechos de los pacientes, el incremento del riesgo legal 
en el ejercicio profesional, así como la mayor aceptación de la idea de que la ciencia y los valores no pueden ser separados ${ }^{(2)}$.

La educación formal en ética médica la definimos como la enseñanza escolarizada de la materia, mediante programas o cursos que forman parte del plan de estudios médicos, las mismas que pueden ser de diversas modalidades: obligatorios o electivos, integrados a otros cursos o independientes. Actualmente, la enseñanza de la ética se ha generalizado y la gran mayoría de las escuelas médicas del mundo tienen en su plan de estudios cursos o programas de formación en ética médica; pero, la experiencia internacional muestra una gran variedad en cuanto a objetivos, contenidos, y metodología.

\section{Experiencia norteamericana}

La educación formal en ética médica tuvo lugar, antes que en ninguna otra parte, en los Estados Unidos de Norteamérica. Veatch y Sollito, en 1974, informaron que $90 \%$ de las escuelas médicas norteamericanas tenía alguna forma de enseñanza de la ética a través de cursos electivos, conferencias, seminarios y otros, mientras que $5 \%$ la había ya establecido como curso obligatorio ${ }^{(3)}$. Para el 2002, 79\% de las escuelas afirmó tener cursos obligatorios de ética médica ${ }^{(4)}$. Eckles y col., en una revisión efectuada en 2004, concluyen que la educación ética es universal en ese país; sin embargo, destacan severas deficiencias en la literatura relacionada con la investigación teórica sobre objetivos, resultados de dicha educación, así como la efectividad de sus métodos de enseñanza ${ }^{(5)}$.

\section{Experiencia británica}

En el marco de su sistema de salud, uno de los más organizados del mundo, la enseñanza ética en las escuelas de medicina británicas cobró impulso con las directrices del General Medical Council (GMC), órgano encargado de regular el ejercicio de la medicina y la educación médica en esa nación que, en 1993, publicó la primera versión de Tomorrow's doctors, documento rector de la educación médica de pregrado, que establece los estándares en materia de objetivos, contenidos, métodos, e incluye aspectos administrativos a los que deben sujetarse equilibradamente las escuelas médicas británicas; dichos estándares incluyen la obligación de incluir cursos de ética y derecho ${ }^{(6)}$. En 1998, la British Medical Journal publicó el artículo Teaching and assessing ethics and law within medical education: a model for the UK core curriculum- Consensus statement by teachers of medical ethics and law in UK medical schools. Este documento fue resultado de un esfuerzo por consensuar entre todos los actores del sistema de salud británico los objetivos, contenidos y métodos que debería tener la enseñanza de la ética en las escuelas de medicina, y es por lo tanto un importante referente mundial ${ }^{(7)}$ periódicamente actualizado; en 2010, se publicó una actualización del citado documento ${ }^{(8)}$.

\section{Experiencia de la Europa continental}

Claudot y col., en un estudio realizado en el 2007, muestran que 24 de las 25 escuelas seleccionadas aleatoriamente de una muestra de 45 tenían alguna forma de enseñanza ética y concluyen que, al igual que en los Estados Unidos, la enseñanza de la ética en Europa presenta grandes diferencias entre sí, debido mayormente a diferencias en recursos de tiempo y medios ${ }^{(9)}$. En España, la implantación de la Bioética como asignatura médica universitaria ha sido facilitada por su pertenencia a la Comunidad Europea y para adecuarse a las directivas de esta, promulgada en 1986, respecto a la enseñanza de la medicina. Pero, se señala insuficiencias: "la clave del retraso que tenemos en la docencia de la asignatura es que escasea la metodología y abunda la confusión conceptual” (10).

\section{Experiencia latinoamericana}

Se viene dando un proceso de incorporación progresiva de la enseñanza de la ética médica, sobre todo a partir de iniciativas individuales de los profesionales de la salud sensibles al tema y a través del estímulo de centros académicos y movimientos sociales en torno a la temática bioética. No obstante, son escasas las publicaciones que den cuenta de este proceso, así como sus características; entre ellas hay quienes señalan que su enseñanza tiene "una visión marcadamente deontológica, que no atiende más a las necesidades exigidas actualmente en la formación del profesional médico" ${ }^{(1)}$; y, en Chile que: "pese a ser una disciplina joven cuenta con un importante desarrollo metodológico y didáctico" (12).

\section{Organización de las Naciones Unidas para la Educación, la Ciencia y la Tecnología (UNESCO)}

Un fuerte estímulo para la enseñanza de la ética y la búsqueda de consensos en cuanto a objetivos, contenidos y metodología proviene de la Declaración Universal sobre Bioética y Derechos Humanos, aprobada en la $33^{a}$ reunión, el 19 de octubre del 2005. Esta Declaración constituye una 'plataforma global común a través de la cual se puede introducir y fomentar la bioética en cada estado miembro'. Define los mínimos (en términos de contenidos y horas) para una formación adecuada en bioética. Puede ponerse en marcha en forma flexible e invita a profesores y estudiantes 'a ampliar sus enfoques y contenidos en distintas direcciones' ${ }^{(13)}$.

\section{Experiencia peruana en educación ética}

La educación formal en ética médica en el Perú se inició en la Facultad de Medicina de la Universidad Nacional Mayor de San Marcos (UNMSM), en 1987, y ha sido incorporada progresivamente en las escuelas de medicina del país. Hoy en día, todas las escuelas consideran, en su plan de estudios, la enseñanza formal de la ética, a través de cursos independientes y/o integrados a otros del área clínica. La Comisión de Acreditación de las Facultades 
Medicina (CAFME), creada por la Ley N 27154, publicó la primera versión de los estándares que debería obligatoriamente cumplir la escuelas de medicina, los que establecían en el punto 10.3 que 'no menos de un $7 \%$ de los créditos deben estar destinados a cursos de ciencias sociales, humanidades y comunicación'; y, detalla los indicadores de las competencias que debe adquirir en este aspecto el estudiante para graduarse ${ }^{(14)}$. En una segunda versión de estos estándares, publicada en el año 2009 por el Consejo Nacional de Evaluación, Acreditación y Certificación de la Calidad de la Educación Universitaria (CONEAU) que sustituyó a la CAFME, la obligatoriedad de la educación en humanidades y por lo tanto en ética no aparecen con el detalle que tenían en la primera versión; ha quedado reducido a una alusión contenida en el ítem 25 que incluye un párrafo que a la letra dice: "instrucción preclínica y clínica de todos los sistemas orgánicos, incluyendo aspectos importantes bioéticos y éticos de prevención, de tratamiento de enfermedades agudas, crónicas, rehabilitación y cuidados de enfermos terminales" ${ }^{(15)}$. Por su lado, la Asociación Peruana de Facultades de Medicina (ASPEFAM), institución representativa de las facultades de medicina del Perú, con rol asesor reconocido por el Estado en materia de educación médica, no considera, entre sus prioridades explicitas, el impulso a la educación formal en ética ${ }^{(16)}$.

\section{Características de la enseñanza ética}

Los aspectos que concitan mayor interés al momento de discutir la implantación de la enseñanza de la ética médica y evaluar su impacto en la formación del médico y su ejercicio profesional son: ¿Para qué enseñar ética médica (objetivos)? ¿Qué enseñar (contenidos)? ¿Cómo y cuándo enseñar y evaluar (métodos)? Adicionalmente, se indaga ¿Quiénes deben enseñar? Diversos enfoques se han formulado para responder a estas preguntas; en lo que sigue, resaltamos aspectos generales para abordar en detalle los diversos aspectos planteados al momento de discutir los resultados de este estudio. En Norteamérica, los bioeticistas más representativos plantean que "el carácter moral básico del estudiante de medicina está ya formado al entrar a la escuela de medicina. El currículo de ética médica no está diseñado para mejorar el carácter moral de los futuros médicos sino para dotar, a quienes ya tienen un buen carácter, de los conocimientos intelectuales y las habilidades de relación que permitan que ese carácter moral se exprese en el mejor comportamiento posible" (17). De otro lado, la perspectiva pedagógica desagrega los tres componentes clave de todo proceso de aprendizaje: conocimientos, habilidades y actitudes. La primera "pueden enseñarse igual que se enseña la anatomía...”. Así, la formación en bioética pretende "la adquisición de actitudes duraderas que mejoren la forma en que los profesionales sanitarios ejercitan su profesión día a día o, en otras palabras, capacitar al participante a transferir teoría y reflexión ética en discernimiento y acción" ${ }^{(18)}$. La mayoría de autores coinciden en que lo único que puede pretenderse en los cursos de ética médica es enseñar conocimientos y habilidades, no directamente actitudes. A ello se suma la necesidad de un enfoque mayéutico en la enseñanza de la bioética ${ }^{(19)}$.

$\mathrm{Al}$ respecto es importante mencionar el aporte de Hafferty y Franks, profesores de la Universidad de Minnesota que, en 1994, publican un artículo especialmente significativo titulado The hidden curriculum, ethics teaching and the structure of medical education, que aborda el acuciante problema de las relaciones entre la enseñanza formal y la informal de la ética que reciben los estudiantes durante su formación en las escuelas de medicina. El enfoque pedagógico orientado por la ética como 'identidad profesional', por el que abogan los autores citados, parte de establecer que todo proceso de educación es una forma de socialización y que como toda socialización tiene una dimensión moral, 'la instrucción formal en ética médica no tiene lugar en un vacío cultural'. Este hecho, aparentemente obvio, es poco reconocido al momento de discutir cómo enseñar la ética y sobretodo no se aprecia en su magnitud el hecho de que la mayor parte de los valores, actitudes, creencias y comportamientos relacionados, considerados importantes en medicina, son internalizados por los estudiantes no a través del currículo formal sino por vía de uno latente, oculto (hidden curriculum), que puede ser más eficaz en replicar la cultura médica que la enseñanza de conocimientos y técnicas ${ }^{(20)}$.

En el Reino Unido existe un amplio acuerdo acerca del perfil ético y legal aceptable para una buena y segura práctica médica, y como se ha dicho, poco desacuerdo acerca de cuáles son los conocimientos, habilidades y actitudes que se requieren para ello. En 1998, se publica una propuesta de un currículo básico ${ }^{(7)}$. Y, una publicación reciente (2010) comunica una actualización del citado consenso. En este se señala como objetivos de la enseñanza: a) capacitar para una buena práctica médica futura; b) desarrollar sensibilidad y comprensión de las responsabilidades éticas, legales y profesionales, necesarios como estudiantes y médicos; c) pensar y reflexionar críticamente sobre temas éticos, legales y profesionales; d) comprender y respetar las fortalezas y debilidades de puntos de vista diferentes de los propios, manteniendo la integridad personal; e) reconocer y responder adecuadamente ante la incertidumbre clínica y ética; f) adquirir conocimiento que facilite la toma de decisión ética y juicio clínico justificable moral, legal y profesionalmente; g) responder adecuadamente ante los nuevos desafíos de la práctica médica resultado de los avances científicos y los cambios sociales; h) integrar conocimientos, habilidades, actitudes y conductas necesarios para la práctica médica profesional ${ }^{(9)}$. La Organización de las Naciones Unidas para la Educación, la Ciencia y la Tecnología (UNESCO) ${ }^{(13)}$ plantea un interesante contenido, valioso por el proceso global de consenso con el que 
fue elaborado, y se fundamenta en los principios. El currículo básico de bioética, a diferencia de otros, incorpora temas vinculados a la responsabilidad de los profesionales de la salud con la sociedad de la que forma parte y los derechos humanos. Estos contenidos son: 1) ¿Qué es la ética?; 2) ¿Qué es la bioética?; 3) Dignidad y derechos humanos; 4) Beneficios y efectos nocivos; 5) Autonomía y responsabilidad individual; 6) Consentimiento; 7) Personas carentes de la capacidad de dar su consentimiento; 8) Respeto de la vulnerabilidad humana y la integridad personal; 9) Privacidad y confidencialidad; 10) Igualdad, justicia y equidad; 11) No discriminación y no estigmatización; 12) Respeto de la diversidad cultural y del pluralismo; 13) Solidaridad y cooperación; 14) Responsabilidad social y salud; 15) Aprovechamiento compartido de los beneficios; 16) Protección de las generaciones futuras; 17) Protección del medio ambiente, la biósfera y la biodiversidad.

Los objetivos del estudio fueron describir las características de la enseñanza ética: nombre, año de inicio y organización del curso (número de horas semanal y total, total de créditos académicos, año de estudio y departamento académico responsable del curso); metodología de enseñanza y evaluación del aprendizaje, objetivos, contenidos y material de lectura, contenidas en los sílabos del curso de ética de las escuelas de medicina peruanas.

\section{MÉTODOS}

Para fines del presente estudio, se solicitó los sílabos del curso de ética a las escuelas. El material lo constituyeron los datos de fuente documental, 25 sílabos de cursos de ética correspondientes a 22 escuelas de medicina peruanas -13 públicas y 9 privadas-, obtenidos el año 2009. Tres escuelas contaban con dos cursos de ética (UNMSM, UNP y UPLA).

Una vez obtenido el material de estudio (25 sílabos) se procedió a:
1) Transcribir los objetivos, contenidos y la lista de referencias bibliográficas de los 25 sílabos en el programa Atlas ti. Se utilizó el análisis de contenido como método para analizar los objetivos (competencias) y los contenidos teóricos del curso ${ }^{(21)}$.

2) Siguiendo el procedimiento para análisis de contenido, se revisó la literatura para establecer criterios de clasificación de objetivos y contenidos del curso. En la revisión se halló documentos de consenso respecto a la enseñanza ética ${ }^{(7,17,22)}$.

La sección Objetivos/competencias del sílabo la denominamos así porque, si bien la mayoría -17 sílabos (68\%) - utilizaba el término competencias, cinco (20\%) utilizaban ambos, objetivos y competencias, dos (8\%) solo objetivos y uno (4\%) el término logros del curso. Se revisó la literatura relevante al tema y se creó las categorías de: actitud/conducta, conocimiento, conocimiento/habilidad, habilidad, sensibilidad, generalidad y otro.

En Contenidos teóricos, se revisó la literatura y se creó las categorías de: misceláneas, ethos profesional, generalidades sobre ética, relación clínica, derecho y ley, vida y muerte, reproducción humana, investigación biomédica, teorías filosóficas, bioética principios, consentimiento informado, genética, servicios de salud, método de análisis de caso.

3) En la prueba de confiabilidad participaron tres investigadores quienes, en forma independiente, asignaron los objetivos y contenidos de los sílabos a las categorías establecidas. Una vez alcanzado un buen nivel de confiabilidad inter-evaluador se procedió a clasificar los objetivos y contenidos de todos los sílabos. Esto se hizo tomando en cuenta las recomendaciones respecto a las técnicas de investigación ${ }^{(23)}$.

Para el análisis estadístico se utilizó el Excel para elaborar la base de datos cuantitativos, y el paquete estadístico SPSS 15 para análisis de frecuencia.

\section{RESULTADOS}

La denominación más frecuente del curso fue Ética y deontología (médica) en diez escuelas y Bioética en cuatro. Cuatro escuelas utilizaban el término de ética médica, solo o con bioética, legislación y sociedad. Dos nombres aludían a preliminares de un segundo curso, Introducción a la Ética y Fundamentos de Ética curso. Las otras denominaciones fueron: Ética, bioética y deontología; Ética y moral social; Ética; Deontología médica; y, Ética y bioética.

El año en que se inició su dictado varió entre 1986 y 2009.

El año de estudio en la que se ubicaba el curso de ética varió entre el primero y el sexto año. En la mayoría de escuelas, 19 (76\%), estaba en la etapa clínica de la educación médica. El año más frecuente fue tercero (ocho escuelas), seguido del quinto (cinco).

La mayoría de escuelas señalaba que la duración del curso era 17 semanas; sin embargo, el total de horas de clase varió entre un máximo de 144 horas a un mínimo de 32 , siendo el promedio 68,36 horas (DE 24).

El número de créditos asignados al curso varió entre 2 y 4 , siendo la media 2,7 (DE 0,56). Tres créditos fue el más frecuente (61\%), seguido de dos $(34,8 \%)$ y solo una escuela le asignó cuatro créditos.

Los departamentos académicos a cargo del curso fueron de lo más variados: ciencias básicas, ciencias biomédicas, ciencias y tecnología social $(\mathrm{n}=$ 7); medicina $(n=5)$; psiquiatría, salud mental $(n=4)$; salud pública, comunitaria $(n=2)$; filosofía, humanidades $(n=2) ; y$, ciencias quirúrgicas $(n=1)$. Cuatro escuelas no contaban con departamento académico.

Los términos usados en los sílabos para referirse al método de enseñanza del curso fueron diversos: sistemas de enseñanza, procedimiento didáctico, metodología, estrategias metodológicas, metodología de aprendizaje, metodología didáctica, metodología de la 
enseñanza y estrategias didácticas. La totalidad de las escuelas consignó clases teóricas con particularidades tales como: con plena (activa) participación del estudiante, dialogada, deliberativo, conferencias y exposiciones, clase magistral dialogada. Las prácticas se refirieron a seminarios, talleres, discusiones, mesas redondas (actividades en aula), mas no práctica clínica con pacientes en vivo.

Los términos usados para referirse a los métodos de evaluación fueron: sistema de evaluación de aprendizaje; indicadores, técnicas e instrumentos de evaluación; forma y herramientas de evaluación; evaluación de la asignatura. La mayoría de escuelas evaluaba aprendizaje de la ética a través de exámenes de conocimiento teórico. Lo que varió fue el porcentaje que asignado al examen, entre 20 y $100 \%$, siendo el promedio 50,5\% (DE 25,3). Quince sílabos señalaban porcentaje de inasistencia que inhabilitaba al alumno a dar examen o aprobar el curso, que varió entre 10 y $30 \%$, siendo el más frecuente $30 \%$.

La tabla 1 presenta las categorías de objetivos/competencias en orden de frecuencia. El objetivo a alcanzar con mayor frecuencia fue el de conocimiento, $42,8 \%$ si sumamos conocimiento $(26,5 \%)$ más conocimiento y habilidad $(16,3 \%)$.

Los contenidos de los sílabos fueron clasificados en 14 categorías y la tabla 2 presenta su orden de frecuencia.

Los 25 sílabos listaban un total de 240 referencias bibliográficas. De ellas, 121 (50,4\%) fueron citas únicas; 45 (18,8\%) citadas dos veces; y 33 (13,8\%) correspondieron a páginas web. La lectura más frecuente (64\%) fue el Código de ética y deontología del Colegio Médico del Perú, seguido del texto de Ética médica de Luis Vélez Correa.

\section{DISCUSIÓN}

Hoy en día, a nivel mundial, la educación ética es una característica regular del currículo de estudios médicos. Sin
Tabla 1. Objetivos/competencias de los silabos.

$\begin{array}{lc}\text { Objetivos/competencias } & \mathrm{n}=344 \\ \%\end{array}$

embargo, la revisión de la literatura evidencia que no existe un modelo único ni tampoco consenso sobre la mejor manera de educar en ética al futuro médico. Así, estudios norteamericanos concluyen que en no existe nada cercano a un currículo común en ética médica en su país ${ }^{(4)}$.

No obstante lo mencionado en la experiencia norteamericana, existen otras tendientes a la búsqueda de consensos respecto al diseño curricular básico en la formación ética de pre-grado y superar la heterogeneidad existente. Este es el caso del Reino Unido, favorecida probablemente por la mayor organización e integración del conjunto de su sistema de salud -el National Health Service- y la preponderancia de instituciones como el General Medical Council, cuyas directrices tienen fuerza normativa. En efecto, en ese país es donde más se ha avanzado en lograr consensos explícitos con la más amplia

participación de escuelas de medicina, instituciones académicas no universitarias, expertos y estudiantes. De ello da cuenta el currículo básico logrado, en $1998^{(7)}$, revisado y actualizado en el $2010^{(8)}$.

El nombre del curso más frecuente en el estudio fue Ética y deontología (médica), seguida de bioética. El Diccionario de la Real Academia de la Lengua Española define 'Ética' como la parte de la filosofía que trata de la moral y de las obligaciones del hombre, y como conjunto de normas morales que rigen la conducta humana; 'Deontología', ciencia o tratado de los deberes; y, 'Bioética', aplicación de la ética a las ciencias de la vida ${ }^{(24)}$. En la literatura de habla inglesa, el término más usual es de 'Ética médica'. Si consideramos que un criterio elemental es que el nombre del curso refleje lo más cercanamente posible la materia de la que trata, los objetivos y el contenido de la educa-

Tabla 2. Contenidos del curso de ética.

\begin{tabular}{lc} 
Contenidos & $\mathrm{n}=450$ \\
Ethos profesional (Acto médico, códigos profesionales) & $\%$ \\
Generalidades (Concepto, definiciones e historia de la ética) & 21,4 \\
Relación clínica (Relación médico paciente, veracidad, confidencialidad) & 12,4 \\
Vida y muerte (Fin de la vida, trasplantes, paciente terminal, eutanasia) & 8,2 \\
Derecho y ley (Derechos humanos, leyes y reglamentos, mala praxis) & 7,5 \\
Reproducción humana (Inicio de la vida, control de la natalidad, aborto) & 7,1 \\
Investigación biomédica & 6,2 \\
Teorías filosóficas (Valor, virtud, contrato social, utilitarismo) & 5,5 \\
Bioética, principios (Autonomía, beneficencia, no maleficencia, justicia) & 4,6 \\
Consentimiento informado (Elementos del Cl, rechazo tratamiento) & 4,6 \\
Genética (Molecular, clonación, consejo, genoma, ingeniería) & 4,2 \\
Servicios de salud (Costos, distribución, racionalización, políticas) & 2,8 \\
\hline
\end{tabular}


ción médica, deberá aceptarse que no se trata solo de enseñar deontología, es decir, los deberes de los médicos compilados usualmente en los códigos de normas de conducta profesional. Abocarse a la enseñanza de la 'Bioética', la denominación de una amplia disciplina que aborda variados tópicos que van desde el estudio de los problemas éticos derivados de la ecología hasta los que plantean la innovación tecnológica en la práctica clínica ${ }^{(25)}$, desbordaría también un currículo básico. Por consiguiente, consideramos que, de lo que se trata es, ni más ni menos, de la enseñanza teórica y práctica de una 'ética aplicada', es decir, de la enseñanza de la ética destinada al ejercicio de la medicina.

Sobre el inicio en la enseñanza del curso, la primera escuela de medicina en introducir formalmente la ética en su plan de estudios fue la UNMSM. El resto de escuelas lo hicieron en la década de los 90; y las nuevas creadas, sin excepción, consideraron la materia desde sus inicios, por las exigencias del Sistema de Acreditación de las Escuelas de Medicina del Perú.

Con relación a la organización del curso, la mayoría de las escuelas de medicina participantes en el estudio solo cuentan con un curso de ética, y solo tres de las 22, con dos cursos. Estas últimas tienen un curso introductorio y de fundamentos en la fase pre-clínica. En las restantes escuelas, la asignatura de ética se ubica con mayor frecuencia en la etapa clínica.

Nuestro estudio confirma la ausencia de enseñanza de la ética en el periodo de internado médico ${ }^{(26)}$. Este hecho resulta relevante, porque esta etapa se caracteriza por intensa práctica clínica del estudiante, y en la que es de esperar confronte numerosas situaciones morales, sin tener espacios docentes donde procesar dichas experiencias.

El conocimiento básico y la habilidad de análisis ético alcanzado por el estudiante en la etapa pre-clínica se materializan con la aplicación del co- nocimiento y juicio en la atención del paciente. Sin embargo, las ventajas de esta dicotomía en la experiencia de aprendizaje, etapa pre-clínica y clínica, no son apoyadas por la evidencia empírica ${ }^{(27) .}$

Respecto a la duración y créditos del curso, en nuestras 22 escuelas de medicina la asignatura de ética es independiente y se le asigna 17 semanas (un semestre académico); y el promedio de horas destinadas para su desarrollo es 68. Tres escuelas cuentan con dos cursos, duplicando así el tiempo asignado a la formación ética.

En el Perú, un alumno de medicina debe haber completado un total promedio de 280 créditos para optar el título de médico cirujano. Ello implica que la formación ética solo ocupa el 1,0\% y $2,0 \%$ (en escuelas con dos cursos) del total de créditos. Esta realidad contrasta con la declaración de las escuelas, de formar buenos médicos, con preparación técnico-científica y humana, que reclaman diversos autores.

En las escuelas médicas norteamericanas que tienen un curso obligatorio, dedican en promedio 29 horas a su dictado, el mismo que se basa en conferencias y discusión en pequeños grupos; utilizan una variedad de métodos, tales como estudios de caso, revisión de artículos empíricos, filosofía moral y recientemente estudios de literatura y humanidades ${ }^{(28)}$.

La disparidad entre las escuelas de medicina peruanas también se refleja en el tipo de Departamento académico responsable del curso: en siete están bajo la responsabilidad de ciencias básicas; dos recae en humanidades y filosofía; y las restantes, en diversas especialidades médicas (salud pública, psiquiatría, medicina, cirugía).

En otras latitudes, el consenso es que la educación ética tiene que ser multidisciplinaria. Una estrategia común es tener un equipo de clínicos y no clínicos que trabajan en colaboración, aunque requiere de un coordinador específico con experiencia profesional y académica relevante, aunque no existen estudios empíricos que evalúen el resultado de la enseñanza por diferentes disciplinas ${ }^{(5)}$.

Respecto al método de enseñanza, en el país la enseñanza ética es a través de clases teóricas, aunque algunas señalan teoría con plena participación de los estudiantes, dialogada, deliberativa. Adicionalmente, se señala prácticas/ discusión de casos, seminarios, talleres, foros, aunque no en todas las escuelas; asimismo, se desconoce el tamaño del grupo para estas actividades. La enseñanza en todas las escuelas es en aula y no en campo clínico. Los métodos utilizados en la enseñanza de la ética en las escuelas médicas del país, vistos en conjunto, son aquellos que se desarrollan en aula y no hay, al parecer, experiencias de enseñanza integrada a la práctica clínica, lo que podría llamarse una enseñanza al lado de la cama del enfermo.

En el Reino Unido, el consenso es que la educación ética debe estar 'centrada en casos', especialmente durante la etapa de la formación clínica. "La discusión de casos sirve a muchos objetivos de la educación ética; enseña sensibilidad hacia los aspectos morales de la medicina, ilustra la aplicación de conceptos humanistas o legales en la práctica médica, y muestra a los médicos actuando como agentes morales responsables" ${ }^{(29)}$. Respecto al tamaño del grupo, el Consensus Statement dice que la clave es que la enseñanza sea clínicamente relevante y graduada al nivel académico y de habilidad de la audiencia al que va dirigida, independientemente del tamaño del grupo ${ }^{(7)}$.

Si bien el consenso es que la enseñanza ética debe estar basada en casos, se critica el método del 'caso trágico', aludiendo a aquellos emblemáticos, complejos y raros porque: "alientan a los estudiantes a generalizar; y lo que es peor, alienta el análisis simplista y rutinario, y prácticas descontextualizadas, que no los ayuda a aprender de la experiencia" (29). Otra propuesta es que los casos deben ser seleccionados 
por los propios estudiantes, a partir de su experiencia personal; asimismo, que la enseñanza debe ser contextualizada y permita abordar los factores externos inmediatos y mediatos (p. ej. el crecimiento de la violencia urbana y familiar hasta lo relativo a organización de los servicios de salud y su financiamiento), que determinan el comportamiento y la toma de decisiones clínicas ${ }^{(30)}$.

En América Latina, se critica el modelo de las clases expositivas, "en los cuales los temas son expuestos por los docentes como si fueran verdades absolutas... En este modelo pasivo de enseñanza los alumnos no son estimulados a reflexionar sobre los diferentes valores morales y a respetar convicciones o creencias personales. Según Edgar Morín, la universidad está habilitando profesionales de cabeza llena, siendo que deberían prepararlos para tener una cabeza lista, pues más importante que la acumulación indiscriminada de información, es su correcta organización para que lo enseñado adquiera sentido" (31).

Sobre el método de evaluación, la mayoría de las escuelas evalúa aprendizaje de la ética a través de un examen de conocimiento, que se promedia con notas de práctica (seminarios, talleres), monografía (investigación) y otras. Es decir, nuestras escuelas cumplen con la recomendación de evaluar formalmente el aprendizaje.

No obstante que la evaluación es el medio para mejorar el currículo, surgen muchas dificultades para evaluar el progreso moral de los estudiantes, por: 1) incertidumbre acerca de los objetivos curriculares; 2) falta de consenso entre los profesores de cómo evaluar. Aspectos como sensibilidad por los valores y la competencia en habilidades de relación no son fácilmente evaluables. Adicionalmente, evaluar competencia no solamente implica adquisición, retención y análisis de información, sino que la compasión, la empatía y el respeto del paciente también es parte de la competencia ${ }^{(32)}$.
Se suele diferenciar los criterios de evaluación en cognoscitivos y no cognoscitivos. Los primeros son casi universales, porque son medidos fácilmente y arbitrariamente utilizando puntajes objetivos. En cambio, los segundos son menos frecuentes, porque son subjetivos y por consiguiente más difíciles de establecer, pese a que pueden ser igualmente importantes para el desarrollo profesional de los estudiantes y para su eventual eficiencia como médicos. Un estudio sobre el tema halló que los criterios no cognoscitivos son mayormente aspectos humanistas, incluyendo: honestidad, conducta profesional, apariencia, respeto de la ley, respeto hacia los demás, confidencialidad, abstención de sustancias, solidaridad y responsabilidad económica ${ }^{(33)}$.

Sobre los objetivos/competencias, en el presente estudio, el objetivo señalado con mayor frecuencia en los sílabos fue el de conocimiento $(42,8 \%)$, siendo menos frecuentes actitud/conducta $(12 \%)$ y habilidades (19\%). Este hallazgo contrasta con la recomendación de la "necesidad de dotar a los médicos de una 'sabiduría práctica' y habilidad para identificar valores en la atención de salud. Si bien hay que proporcionarles una base de conocimiento filosófico, social y legal, este es para utilizarlo en el razonamiento clínico; asimismo, hay que dotarlos de las habilidades de relación necesarias para aplicar ese conocimiento en la atención de salud de seres humanos" (32).

En los Estados Unidos, los objetivos hallados en 58 sílabos de ética médica fueron: 1) Familiarizar con tópico de ética médica (p.ej., consentimiento informado, veracidad, futilidad) $(77,6 \%)$; 2) Desarrollar habilidades de razonamiento/solución de problemas éticos $(63,8 \%)$; 3) Aprender teorías normativas/conceptos éticos (34,5\%); Aprender acerca de derecho y medicina $(32,8 \%)$; 5) Promover virtudes médicas e incorporar valores profesionales $(29,3 \%)^{(4)}$.

En el Reino Unido, la General Council of Medicine (GMC), en Tomorrows' Doctors, señala que al finalizar la carre- ra, el estudiante "debe haber alcanzando un conocimiento y comprensión de temas éticos y legales relevantes para su ejercicio profesional, y la habilidad para comprender y analizar problemas éticos así como garantizar a pacientes, sus familiares y la sociedad en general, que observará correctamente tales problemas en la decisión médica" ${ }^{(7)}$.

Una revisión resume el consenso respecto a los objetivos de la educación en ética médica, como que ella es un medio para: 1) dotar a los médicos de un conjunto de habilidades para analizar y resolver problemas éticos; 2 ) crear médicos virtuosos ${ }^{(5)}$. Respecto a esto último, hay opiniones que para crear un médico virtuoso se debe empezar con un sujeto virtuoso. Los comités de admisión de las escuelas que realmente quieren producir médicos orientados éticamente, deben buscar aquellos postulantes que sean mejores ejemplos de virtud. Estos rasgos del carácter han sido señalados por diversos autores e incluyen: fidelidad a la confianza, benevolencia, honestidad intelectual, valor, compasión y confiabilidad ${ }^{(34)}$.

En Contenidos, en nuestro estudio destaca la frecuencia alta de la categoría denominada ethos profesional (conducta médica, profesionalismo), 21,4\%. Esta alude a la ética médica clásica, vigente desde los inicios de la medicina occidental hace 25 siglos. Es la ética hipocrática que se enseñaba con el ejemplo, el cumplimiento del deber que obliga la profesión, frente al paciente, su familia y la sociedad. Sin embargo, los temas de la nueva ética, que surge con la medicina moderna, p. ej., el consentimiento informado $(4,2 \%)$ y la justicia sanitaria $(1,5 \%)$, no se les enseña en todas las escuelas. Respecto al consentimiento, el que no se enseñe en varias escuelas médicas peruanas explicaría la frecuencia baja de aciertos en un examen de conocimiento sobre el tema ${ }^{(35)}$; asimismo, el segundo tema, pese a que casi no es tocado en la asignatura de ética, fue identificado como el problema ético más frecuente en la experiencia clínica de médicos jóvenes ${ }^{(36)}$. 
En los Estados Unidos, el estudio de 58 sílabos halló seis áreas de contenido que se enseña en la mayoría de las escuelas: consentimiento informado $(85 \%)$; servicios de salud (75\%); intimidad y confidencialidad (67\%); calidad de vida/futilidad (67\%); muerte y morir $(66 \%)$; eutanasia y suicidio asistido $(60 \%)$. Señalan el hallazgo de evidentes insuficiencias o inadecuaciones entre lo que se enseña y las previsibles necesidades en el ejercicio profesional, p. ej., falencia de temas relacionados con los comités y consultoría ética, los exámenes genéticos ya comunes actualmente y que serán más frecuentes en el futuro ${ }^{(4)}$.

En otro estudio en los EE UU y Canadá se halló: consentimiento informado (96\%); fin de la vida (92\%); confidencialidad (92\%); veracidad en la información (90\%); ética del estatus de estudiante $(75 \%)$; y distribución de escasos recursos $(75 \%){ }^{(28)}$.

El foco de la ética médica se ha ampliado, desde los dilemas clásicos y la determinación de lo que es ético, hasta examinar conducta ética bajo las limitaciones de la práctica médica actual, dando mayor énfasis a temas rutinarios ('ética cotidiana'). Otra dimensión de esta ética es su sustento en temas no controversiales, porque educar en ética no implica enseñar dilemas y mucho menos los insolubles. Y, en su lugar, hay que transmitir mensajes precisos basados en principios y valores de consenso ${ }^{(27)}$.

En nuestro estudio llamó la atención la amplia variedad de referencias bibliográficas listadas en los sílabos de ética $(n=250)$, de los cuales $50 \%$ fueron citadas por una sola escuela y $19 \%$ por dos. Entre las referencias citadas con mayor frecuencia está el Código de ética y deontología del Colegio Médico del Perú (64\%) seguido del texto de Ética médica de Luís A. Vélez Correa (56\%). Este hallazgo corrobora, de un lado la heterogeneidad de los sílabos en general y del material bibliográfico en particular; y, de otro, la coincidencia entre las escuelas en el uso de un docu- mento deontológico y un texto de ética médica tradicional. Adicionalmente, entre las fuentes bibliográficas es llamativa la ausencia de los esfuerzos editoriales más regulares de la región, nos referimos al Acta Bioética, publicación de la Oficina de Bioética de la Organización Panamericana de la Salud.

En los Estados Unidos, el estudio de 58 sílabos listaban un total de 1191 referencias bibliográficas, entre obligatorias y recomendadas, y solamente ocho de ellas fueron utilizadas por más de seis escuelas. La primera fuente, en orden de frecuencia fue: Beuchamp TL y Childress JF. Principles of Biomedical Ethics 4th edition. Oxford. New York, 1994; seguida de, Lo B. Resolving Ethical Dilemas: a Guide for Clinicians. Williams \& Wilkins. Baltimore, $1995^{(4)}$. Este estudio y el nuestro evidencia, de un lado, la falta de consenso en cuanto a fuentes bibliográficas; y, de otro, la escasez de textos de ética médica en la formación de pre-grado.

Las limitaciones del presente estudio fueron dos: primero, las dificultades en el análisis de los sílabos, debido a su heterogeneidad. Se tuvo que recurrir a prueba de confiabilidad interevaluador para superar el obstáculo. En segundo lugar, nuestro estudio sobre la educación en ética médica no recoge la opinión de autoridades ni profesores de las escuelas de medicina. Al respecto, creemos importante citar los estudios en profesores de la Facultad de Medicina de la UNMSM y de dos hospitales públicos, ambos citados en la experiencia peruana en educación ética ${ }^{(37)}$.

\section{AGRADECIMIENTO}

A las escuelas de medicina participantes y personas que hicieron posible la realización del presente estudio.

\section{REFERENCIAS BIBLIOGRÁFICAS}

1. Gracia D. La bioética, una nueva disciplina académica. JANO. 1987;33(781):69-74.

2. Gracia D. Fundamentos de Bioética. Madrid: EUDEMA S. A. 1989:11.
3. Veatch R, Sollito S. Medical ethics teaching. Report of a National Medical School. JAMA. 1976;235:1030-3

4. DuBois JM, Burkemper J. Ethics education in US medical schools: a study of syllabi. Acad Med. 2002;77:432-7.

5. Eckles RE, Meslin EM, Gaffney M, Helft PR. Medical ethics education: where we are? Where should we be going? A review. Acad Med. 2005;80:1143-52.

6. General Medical Council. Tomorrow's doctors. Recommendations undergraduate medical education, issued by the Education Commitee of the General Medical Council in pursuance of section 5 of the Medical Act 1983. December 1993.

7. Consensus statement by teachers of medical ethics and law in UK medical schools. Teaching and assessing ethics and law within medical education: a model for the UK core curriculum. J Med Ethics. 1998;24:188-92.

8. Stirrat GM, Johnston C, Gillon R, Boyd K; on behalf of the Medical Education Working Group of the Institute of Medical Ethics and associates signatories. Medical ethics and law for doctors of tomorrow: the 1998 Consensus Statement updated. J Med Ethics. 2010;36:55-60.

9. Claudot F, Alla F, Ducrocq X, Coudane H. Teaching ethics in Europe. J Med Ethics. 2007; 33(8):491-5.

10. Altisent R. Experiencias metodológicas ante el reto de la enseñanza de la ética. Educación Médica. 2006;9 (Supl 1).

11. de Siqueira 2008 www.saber.ula.ve/ bitstream/123456789/23754/2/articulo3.pdf

12. León F. Enseñar bioética: como trasmitir conocimientos, actitudes y valores. Acta Bioética. 2008;14(1).

13. UNESCO Bioethics Core CurricuIum 2008. http://unesdoc.unesco.org/ images/0016/001636/163613e.pdf.

14. Comisión para la Acreditación de Facultades o Escuelas de Medicina. Ley, Reglamento, Estándares mínimos de Acreditación y Manual de procedimientos. Enero 2002.

15. Consejo de Evaluación, Acreditación y Certificación de la calidad de la Educación Superior Universitaria (CONEAU). Compendio Técnico Normativo. Tomo V. Estándares para la Acreditación de las carreras profesionales universitarias de Medicina, Enfermeria y Obstetricia. Lima-Perú. 2010.

16. ASPEFAM. III Congreso Peruano de Educación Médica. Declaración de Lima, Octubre 2010. Página web ASPEFAM.

17. Culver ChM, Clouser KD, Pert B, Brody H, Fletcher J, Jonsen A, Kopelman L, Lynn J, Siegler M, Wikler D. Special Report. Basic curricular goals in medical ethics. N Engl J Med. 1985;312(4):253-6.

18. Asociación de Bioética Fundamental y Clínica. Grupo de trabajo sobre la enseñanza de la bioética. La educación en bioética de los profesionales sanitarios en España: una propuesta de organización. Madrid: Ed. Lormo, 1999.

19. Gracia D. La enseñanza de la bioética en España: un enfoque socrático. En fundamentación y enseñanza de la bioética, 2da edición. Bogotá: Editorial El Buho. 2000.

20. Hafferty FW, Franks R. The hidden curriculum, ethics teaching, and the structure of medical education. Acad Med. 1994;69:861-71.

21. Triviños A. Introducción a la investigación en Ciencias Sociales Primera Edición. Sao Paulo: Altas. 1990. 
22. A Working Group on Behalf of the Association of Teachers of Ethics and Law in Australian and New Zealand Medical School (ATEAM). An ethics core curriculum for Australian Medical School. MJA. 2001;175:205-10.

23. Goode WJ, Hatt PK. Métodos de investigación social. México: Editorial Trillas. 1988:382 pp.

24. Diccionario de la lengua española. Vigésima edición. Disponible en: http://lema.rae.es/drae/

25. Rivero P, Pérez RI. Ética y bioética. En: Pérez Tamayo R, Risker R, Tapia R (coordinadores). En la construcción de la bioética. México: Fondo de Cultura Económica. 2007.

26. Alarcón J, Piscoya J, Gutiérrez C, Reyes M. Enseñanza de bioética en las escuelas de medicina del Perú. An Fac med. 2005;66(1):S58-59.

27. Goldie J. Review of ethics curricula in undergraduate medical education. Medical Education. 2000;34:108-19.

28. Lehmann LS, Kasoff WS, Koch P, Federman DD. A survey of medical ethics education at US and Canadian medical schools. Acad Med. 2004;79:682-9.
29. Liaschenko J, Oguz NY, Brunnquell D. Critique of the "tragic case" method in ethics education. J Med Ethics. 2006;32:672-7.

30. Fox E, Arnold RM, Brody B. Medical ethics education: past, present and future. Acad Med. 1995;70:761-9.

31. Siqueira JE. Educación en bioética. En: Tealdi JC (Director). Diccionario Latinoamericano de Bioética. UNESCO: RedBioética, Universidad Nacional de Colombia.

32. Miles SH, Lane LW, Bickel J, Walter RM, Casse ChK. Medical ethics education: coming of age. Acad Med. 1989;64:707-14

33. Miller GD, Frank D, Franks RD, Getto CJ. Non cognitive criteria for assessing students in North American medical schools. Acad Med. 1989;64:42-5.

34. Lakhan SE, Hamlat E, McNamee T, Laird C. Time for a unified approach to medical ethics. Philosophy, Ethics and Humanities in Medicine. 2009;4:13.

35. Zavala S, Sogi C, Delgado A, Cárdenas M. Conocimiento sobre consentimiento informa- do de médicos en formación. An Fac med. 2010;71(2):103-10.

36. Sogi C, Zavala S, Delgado A, Cárdenas, M. Experiencia con problemas éticos en la práctica clínica de médicos jóvenes peruanos. Acta méd peruana. 2010:27(4):227-32.

37. Perales A. Ética y humanismo en la formación Médica. Acta Bioética. 2008;14(1):30-8.

Artículo recibido el 19 de setiembre de 2012 y aceptado para publicación el 2 de febrero de 2013.

Correspondencia:

Dr. Maximiliano Cárdenas Díaz

Correo electrónico: maxcdiaz@yahoo.es 